\title{
Cordoma en la articulación facetaria de C4: reporte de caso
}

\section{Chordoma in C4 Facet Joint: A Case Report}

Fecha de recepción: 14 Marzo 2016 | Fecha de aprobación: 24 Marzo 2017

\author{
María Gabriela Calvo V. \\ Pontificia Universidad Javeriana, Colombia \\ Juan Carlos Acevedo González ${ }^{\mathrm{a}}$ \\ Pontificia Universidad Javeriana, Colombia \\ Miguel Enrique Berbeo C. \\ Pontificia Universidad Javeriana, Colombia
}

a Autor de correspondencia. Correo electrónico: jacevedog@gmail.com

\section{RESUMEN}

Los cordomas son tumores óseos primarios, poco frecuentes, derivados de remanentes no diferenciados de la notocorda. Por su origen histológico, suelen ubicarse en la línea media del esqueleto axial, y los lugares de presentación más frecuentes son la base del cráneo y la columna. Se caracterizan por presentar un crecimiento lento, por lo que tienden a ser clínicamente silenciosos hasta alcanzar tamaños que causan manifestaciones que varían según el sitio de presentación; sin embargo, tienen alta agresividad y recurrencia local. El tratamiento es quirúrgico e, idealmente, se busca una resección completa de la lesión. El artículo presenta el caso de un hombre de 20 años de edad, quien desarrolló un cordoma en la articulación facetaria superior izquierda de $\mathrm{C} 4$, que es una localización rara.

Palabras clave

articulación; faceta; tumor; cordoma; dolor.

\begin{abstract}
Chordoma are rare primary bone tumours derived from non-differentiated remains of the notochord. Due to their histological origins, the most common site of presentation is on the mid-line of the axial skeleton, with a distribution that is most frequent on the sacral bone, skull base and mobile spine. These tumours have a slow growth rate, which means that symptoms occur when the size of the mass causes different manifestations according to its site of presentation. However, they have aggressive behaviour with high rates of local recurrence. Ideal treatment is based on surgical block removal if possible. This article presents the case of a 20 year-old male patient with diagnosis of a chordoma on the left superior facetary articulation of $\mathrm{C} 4$.

Keywords

joint; zygapophyseal; tumour; chordoma; pain.
\end{abstract}

\section{Introducción}

En 1856, Hubert von Luschka realizó la primera descripción de un cordoma en el clivus; sin embargo, Rudolf Virchow, 
en 1857, hizo su primera descripción histológica y atribuyó su crecimiento a un origen cartilaginoso con degeneración hidrópica celular. A ello le dio el nombre de células fisaliferas. Estas células se caracterizan por que presentan núcleos grandes con vacuolas intracelulares, un hallazgo característico hoy en día del cordoma. Johannes Peter Müller, en 1858, atribuyó por primera vez el posible origen del tumor a un remanente notocordal, y Hugo Ribbert, en 1894, acuñó el término cordoma, por la teoría notocordal, y desde entonces más de mil casos se han reportado en la literatura [1].

Los cordomas son neoplasias poco frecuentes responsables de $1-4 \%$ de todas las lesiones malignas del hueso y constituyen menos del 5 $\%$ de los tumores en la columna vertebral [2]. Histológicamente, son considerados neoplasias de bajo grado, pero al ser altamente recurrentes y agresivas en el área local, tienen consideraciones similares a tumores de mayor malignidad. Su pronóstico está dado por esa recurrencia local, más que por metástasis a distancia [3].

\section{Caso clínico}

El caso corresponde a un hombre de 20 años de edad, natural y procedente de Bogotá, Colombia. Posee lateralidad diestra y es estudiante universitario.

\section{Enfermedad actual}

El paciente presenta un cuadro clínico de un año de evolución, consistente en dolor en la región cervical de predominio izquierdo, de características mecánicas y que se exacerba ante movimientos de rotación y flexión del cuello. Refiere posteriormente sensación de masa en la región cervical con palpación dolorosa y crecimiento. Tiene incremento progresivo del dolor en intensidad y duración hasta hacerse continuo y llegar a una Escala Numérica Visual de 8/10. El dolor interrumpe el sueño. No refiere síntomas de tipo neuropático. El paciente recibió inicialmente tratamiento con analgésicos y terapia física; pero ante la persistencia del dolor, una valoración por ortopedia permite diagnosticar una masa en la articulación facetaria izquierda de C4. Fue llevado a cirugía en otra institución, y por los hallazgos fue enviado al Hospital Universitario San Ignacio.

\section{Antecedentes}

Patológicos: parotiditis.

Farmacológicos: consumo ocasional de ibuprofeno y acetaminofén.

Tóxico-alérgicos: fumador ocasional desde hace 2 años.

Familiares: Cáncer de tiroides en abuela paterna. Abuela materna: hipertensión arterial y diabetes mellitus tipo 2 .

\section{Examen físico}

Paciente en buen estado general, alerta y orientado. Pares craneales conservados. Fuerza $5 / 5$ en las cuatro extremidades. Reflejos músculo-tendinosos: $++/++++$. Sin signos de mielopatía o radiculopatía. Sin dolor neuropático. Respuesta plantar flexora bilateral. Sin signos meníngeos. Se observa una cicatriz antigua en buen estado en el dorso de la región cervical izquierda. 
Estudios imagenológicos

\section{Imágenes prequirúrgicas}

Figura 1.Tomografía axial computarizada en proyecciones coronal, sagital y axial que muestran una lesión expansiva de aspecto lítico que afecta la faceta articular superior izquierda de $\mathrm{C} 4$
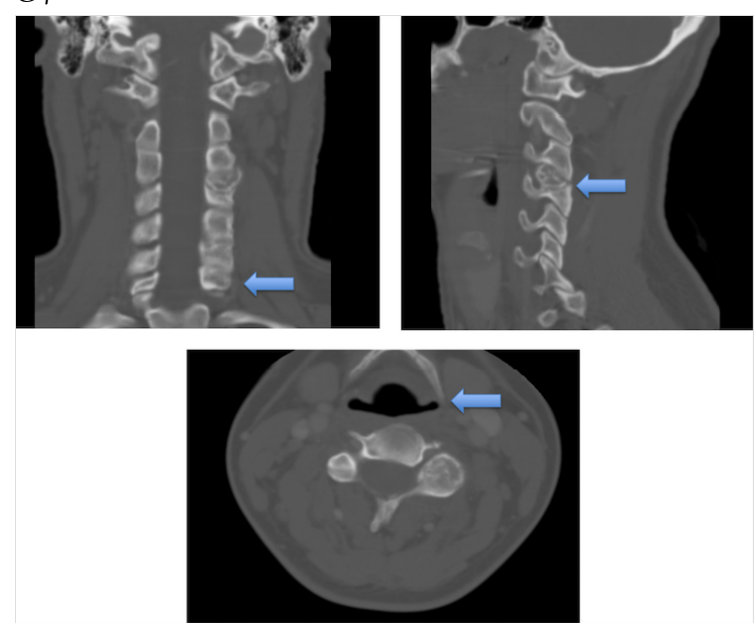

Figura 2.Resonancia magnética nuclear en T2: corte sagital y axial. Se evidencia una lesión hiperintensa con irregularidad ósea del proceso articular izquierdo de $\mathrm{C} 4$

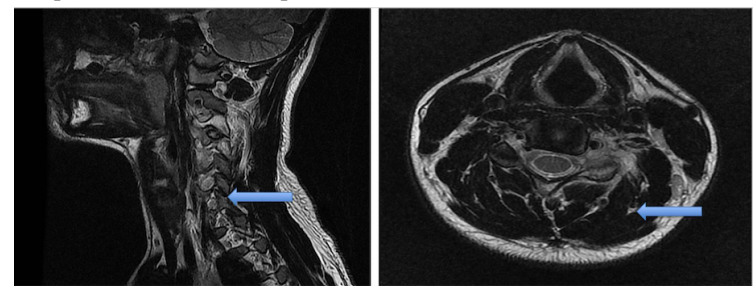

\section{Estudio histopatológico}

Lesión de aspecto mixoide donde se observan células grandes epitelioides con citoplasma vacuolado y eosinofílico. La muestra fue reactiva para proteína S-100 y negativa para CEA. Adicionalmente, el índice de proliferación fue bajo, menor del $2 \%$. Todos fueron hallazgos compatibles con cordoma (figura 3 ).
Figura 3. A) Tinción con H-E con lesión de aspecto mixoide con células grandes y citoplasma eosinofilico. B) Inmunohistoquímica positiva para proteína S-100. C) Muestra que evidencia un indice de proliferación menor al $2 \%$

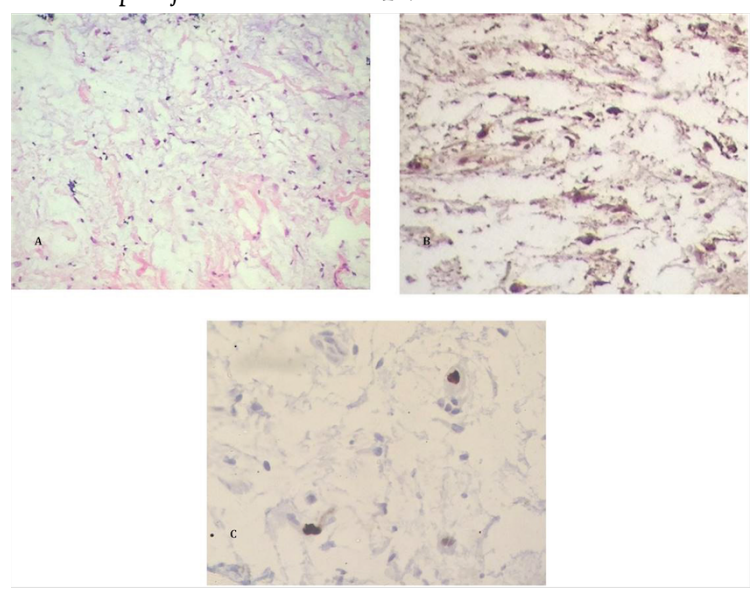

Resonancia magnética posquirúrgica (después de cirugía extrainstitucional)

Las imágenes tomadas revelan cambios posquirúrgicos de resección de masa en la apófisis articular superior izquierda de C4. Se observó una lesión expansiva de señal intermedia en $\mathrm{T} 1$, con realce algo heterogéneo con gadolinio, con medidas aproximadas de $14 \times$ $16 \times 17,5 \mathrm{~mm}$, considerada primera posibilidad residuo o recidiva de la lesión. No se detalló alteración en la amplitud del canal espinal, con cuerpos vertebrales y discos intervertebrales sin alteraciones.

La imagen en T2 evidenció un aumento en la intensidad de señal de los músculos paravertebrales izquierdos entre C3-C4 y C4C5, atribuidos a cambios por el procedimiento quirúrgico, relativamente reciente en el momento de toma de la imagen (figuras 4 y 5 ). 
Figura 4.Corte sagital en T1 y T2. Se observan cambios posquirúrgicos por resección en la apófisis articular superior izquierda de C4
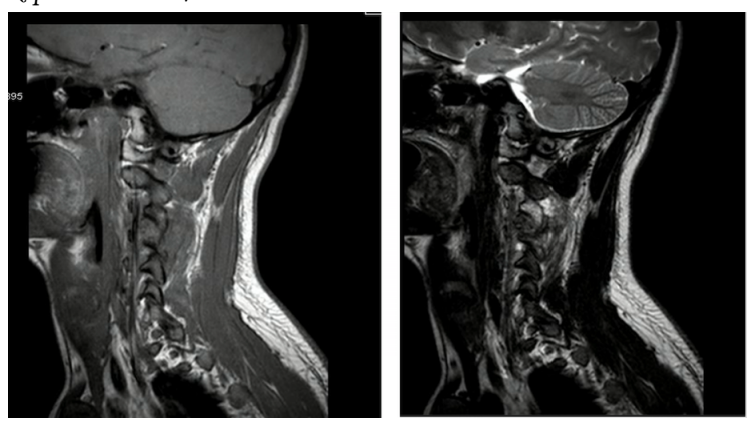

Figura 5.Corte sagital y axial donde se observa realce heterogéneo luego de la inyección con gadolinio. Se evidencia una lesión expansiva de $14 \times 16 \times 17,5 \mathrm{~mm}$
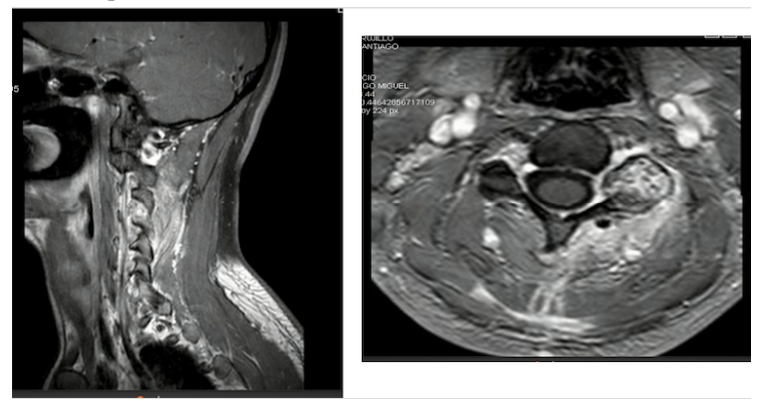

\section{Evolución y tratamiento}

Debido a los hallazgos encontrados en la resonancia magnética posquirúrgica, compatibles con residuo de la lesión tumoral, se realizó un nuevo tiempo quirúrgico con facetectomía de C4 izquierda y resección del tumor, con abordaje posterolateral. Se llevó a cabo una artrodesis posterior de C2-C5. Una vez hecha la intervención, hubo manejo conjunto con oncología para inicio de radioterapia.

\section{Discusión}

Dentro de la epidemiología de los cordomas, estudios de población basados en el Sistema de Vigilancia y Epidemiología, en Estados Unidos [1], han evidenciado una incidencia de 0,8 por cada 100.000 individuos, más predominante en hombres, con una relación de 2:1. Los cordomas tienen un pico de incidencia en personas entre los
50 y los 60 años, y son raros en personas menores de 40 años. Los casos en niños y adolescentes representan menos del $5 \%$ de todos los cordomas [1].

Históricamente, la distribución anatómica del cordoma se ha descrito más frecuentemente en la región sacra $(60 \%)$ y en la base de cráneo $(25 \%)[2,3,4,5]$. Su aparición en la columna móvil representa el $15 \%$, con una distribución del $67 \%$ en la columna lumbar; un $28 \%$ en la columna cervical, y un $11 \%$ en la columna torácica [3]. Dicha descripción se encuentra basada principalmente en el análisis de 262 pacientes de la Clínica Mayo [6], y coincide con registros de pequeñas series en diferentes regiones [3, 4]. Sin embargo, el estudio realizado entre 1973 y 1995, de 400 pacientes en Estados Unidos por medio del Sistema de Vigilancia y Epidemiología, mostró una distribución casi igual entre la aparición en los diferentes segmentos. Se encontró un 32,8\% en la columna móvil, un 32 \% en la base del cráneo y un 29,2 \% a nivel sacro [1].

En la columna móvil se ha descrito que la mayoría de casos ocurren en el cuerpo vertebral y suele expandirse al resto de la vértebra [4]. Se ha observado que nidos de células notocordales corresponden topográficamente con los lugares de ocurrencia del cordoma, lo que explica su localización típica en la línea media $[2,7,8,9,10$, 11]. Sin embargo, el reporte de casos de cordomas extraxiales [12] y como masas paravertebrales sin afectación ósea $[8,9]$ permiten pensar en la existencia de restos ectópicos de la notocorda que expliquen su aparición.

Adicionalmente, la aparición de cordomas en la parte lateral de la base de cráneo se ha explicado por la división en la terminación rostral de la notocorda embrionaria [10]. La revisión de las bases de datos no permitió encontrar ningún artículo donde se presente afectación ósea exclusivamente de la articulación facetaria, cuyo origen sea un cordoma. Los reportes de casos de cordomas extraxiales, por lo general, no tienen compromiso óseo.

Histológicamente, se describe, sobre todo, el cordoma clásico, el condroide y el desdiferenciado. El cordoma clásico suele 
presentarse macroscópicamente como un tumor blando, grisáceo y lobulado que, microscópicamente, está compuesto por grupos celulares en lóbulos separados por tabiques fibrosos. Las células observadas son de gran tamaño, con núcleos redondeados de citoplasma vacuolado descritas como células fisalíferas. El cordoma condroide, además de las características descritas para un cordoma, tiene hallazgos similares a un condrosarcoma.

En el plano inmunohistoquímico, presentan reactividad por la proteína S-100 y marcadores epiteliales, como el antígeno de membrana epitelial y citoqueratinas [2]. Clínicamente, se presentan como tumores indolentes, y por su lento crecimiento suelen ser silentes hasta estadios avanzados, cuando alcanzan grandes tamaños que generan desplazamiento o compresión de estructuras vecinas. La clínica particular cursa con dolor como síntoma principal y déficit neurológico de acuerdo con el lugar de origen.

Aquellos en la base de cráneo suelen presentar una afectación de los pares craneales. Entre tanto, en la columna sacra, la principal manifestación es dolor localizado con raro déficit neurológico. En las zonas cervicales altas o bajas suele aparecer dolor, causado por destrucción ósea, compresión nerviosa o movilidad aumentada del segmento vertebral [5]. $\mathrm{Si}$ presentan un crecimiento anterior, pueden manifestarse con disfagia u obstrucción de la vía aérea e, incluso, como una masa orofaríngea. El crecimiento hacia la zona posterior puede variar desde radiculopatía hasta compresión medular.

Radiológicamente, los cordomas se observan como lesiones solitarias de la línea media, destructoras de hueso. La resonancia magnética es ideal para su diagnóstico, con hallazgos que en T1 suelen verse como lesiones isohipointensas; mientras que en $\mathrm{T} 2$ se ven hiperintensas y con realce con gadolinio [11]. La resonancia magnética permite valorar la extensión a tejidos blandos o estructuras vecinas, y su relación con estructuras anatómicas. La tomografía axial computarizada muestra afectación ósea con imágenes de una lesión ósea lítica que puede tener un componente mixto sólido-líquido con calcificaciones en un $30-70 \%$ de los casos, por secuestro del hueso [12,13].

La cirugía es el pilar del tratamiento. El objetivo es remover el tumor tanto como sea posible, sin causar secuelas, idealmente logrando una escisión en bloque si el lugar de origen lo permite. Para cordomas de columna cervical, la aproximación quirúrgica suele ser anterolateral, si el tumor se limita al cuerpo vertebral, incluso doble (anterior y posterior) si hay extensión al arco posterior. En el caso presentado, dado el compromiso de la articulación facetaria, sin afectación de las estructuras anteriores, se propuso un abordaje quirúrgico por vía posterolateral. Debido a la inestabilidad que es causada durante la intervención quirúrgica, se debe reconstruir y estabilizar la columna.

Se ha visto que la recurrencia del tumor es dos veces mayor cuando la resección en bloque del tumor afecta la cápsula en aquellas intervenciones en que se logra la resección sin afectar la cápsula [6].

La radioterapia postoperatoria suele mejorar la sobrevida; sin embargo, el riesgo de recurrencia está limitado a la calidad de la resección. Idealmente, se debe hacer la escisión quirúrgica seguida de radioterapia con altas dosis, pero el lograr la resección en bloque completa con márgenes libres es suficiente. La tasa de supervivencia varía desde 6 a 29 años, con un porcentaje del $67,6 \%$ a 5 años; del $39,9 \%$ a 10 años, y 13,1 \% a 20 años [6,14].

\section{Conclusiones}

Los cordomas son tumores óseos primarios poco frecuentes, cuya presentación en la línea media sobre el esqueleto axial es la más frecuente; sin embargo, la presentación fuera de la línea media, como en el caso reportado, puede ser explicada por remanentes ectópicos de la notocorda. $\mathrm{Su}$ lento crecimiento genera sintomatología tardía y el diagnóstico imagenológico se realiza idealmente por resonancia magnética. La cirugía es el tratamiento de elección con posterior radioterapia si no se logra escisión con bordes 
libres. El pronóstico está dado por la recurrencia local más que por metástasis a distancia.

\section{Referencias}

1. McMaster M, Goldstein A, Bromley C, Ishibe N, Parry D. Chordoma: incidence and survival patterns in the United States, 1973-1995. Cancer Causes Control. 2001;12:1-11.

2. Walcott B, Nahed B, et al. Chordoma: Current concepts, management, and future directions. Lancet Oncol. 2012;13:e69-76.

3. D'Haen B, Jaegere T, Goffin J, Dom R, et al. Case report: Chordoma of the lower cervical spine. Clin Neurol Neurosurg. 1995;97(6):245-8.

4. Boriani S, Bandiera S, Biagini $\mathrm{R}$, et al. Chordoma of the mobile spine: Fifty years of experience. Spine. 2006;31(4):493-503.

5. Chihani M, Darouassi Y, Touati M, Ammar $\mathrm{H}$, Bouaity. Chordoma of the cervical spine: a case report and review of literature. $\mathrm{Br} \mathrm{J}$ Med Med Res. 2015;5(7):955-61.

6. Jiang L, Liu ZJ, Liu XG, Ma QJ, Wei F, Lv Y, et al. Upper cervical spine chordoma of $\mathrm{C} 2$ C3. Eur Spine J. 2009;18:293-300.

7. Lantos J, Agaram NP, Healey JH, Hwang S. Recurrent skeletal extraaxial chordoma confirmed with brachury: imaging features and review. Skeletal Radiol. 2013;42:1451-9.

8. Bannur U, Chawda SJ, O'Donovan DG, Kaddour H, David KM. Paravertebral cervical chordoma-a case report. $\mathrm{Br} \mathrm{J}$ Neurosurg. 2011;25(4):527-9.

9. Matsubayashi J, Sato E, Nomura M, Kakihana M, Uchida O, Saji $\mathrm{H}$, et al. A case of paravertebral mediastinal chordoma without bone destruction. Skeletal Radiol. 2012;41:1641-4.

10. Kaufman BA, Francel PC, Roberts RL, Argemand E, Park TS, Dehner LP. Chondroid chordoma of the lateral skull base. Pediatr Neurosurg. 1995;23(3):159-65.
11. Wippold F, Koeller K, Smirniotopoulos J. Clinical and imaging features of cervical chordoma. Am J Radiol. 1999;172:1423-6.

12. Romera C, Wiehoff A, Pérez V, et al. Cordoma cervical: a propósito de un caso. Radiología. 2002;44(2):65-8.

13. Hyun S, Rhim SC, Riew D. A combined posterior, lateral, and anterior approach to ventrolaterally situated chordoma of the upper cervical spine. Surg Neurol. 2009;72:409-13.

14. Mühlbauer M, Knosp E. The lateral transfacetal retrovascular approach for an anteriorly located chordoma originating from the second cervical vertebra. Acta Neurochir. 2001;143(4):369-76. 